International Journal of Pure and Applied Mathematics

Volume 97 No. 2 2014, 147-160

ISSN: 1311-8080 (printed version); ISSN: 1314-3395 (on-line version)

url: http://www.ijpam.eu

doi: http://dx.doi.org/10.12732/ijpam.v97i2.3

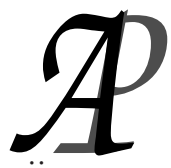

ijpam.eu

\title{
SECOND ORDER DIFFERENTIAL-DIFFERENCE EQUATION FOR DUNKL-CLASSICAL ORTHOGONAL POLYNOMIALS
}

\author{
J. Alaya ${ }^{1}$, B. Bouras ${ }^{2} \S$, Y. Habbachi ${ }^{3}$ \\ ${ }^{1,2}$ Department of Mathematics \\ College of Sciences \\ Qassim University \\ KINGDOM OF SAUDI ARABIA \\ ${ }^{3}$ Higher Institute of Applied Mathematics \\ and Informatics of Kairouan \\ TUNISIA
}

\begin{abstract}
In this paper, we show that Dunkl-classical polynomial sequences can be characterized by a differential-difference equation.
\end{abstract}

AMS Subject Classification: 33C45, 42C05

Key Words: orthogonal polynomials, Dunkl-classical polynomials, regular forms, D-semiclassical polynomials

\section{Introduction}

Linear second order difference and differential equations with polynomial coefficients appear in many research domains such as atomic, molecular and nuclear physics, electrodynamics and acoustics. The links between them and classical orthogonal polynomials are extensively studied by many authors. For instance,

Received: February 11, 2014

(C) 2014 Academic Publications, Ltd. url: www.acadpubl.eu

${ }^{\S}$ Correspondence author 
we know since Bochner [2] that classical orthogonal polynomials of continuous variables are characterized by some second order differential equations. The literature on this topic is extremely vast. We quote for instance [8]-[11]. This characterization has been extended for q-classical orthogonal polynomials to a second order q-difference equation (see [7], [14]). Analogously, classical orthogonal polynomials of discrete variables were characterized by a linear second order difference equation [6].

A naturel question arises: Is there a similar characterization for Dunklclassical polynomials?

Recall that a monic orthogonal polynomial sequence $\left\{P_{n}\right\}_{n \geq 0}$ is called Dunkl-classical polynomial sequence (the associated form is called Dunkl-classical form) if $\left\{T_{\mu} P_{n}\right\}_{n \geq 1}$ is an orthogonal polynomial sequence, where $T_{\mu}$ is the Dunkl operator [5] defined by $T_{\mu}=D+2 \mu H_{-1}$, whith $\mu>-\frac{1}{2}$ and $D$ (resp. $H_{-1}$ ) denotes the derivative operator $D=\frac{d}{d x}$ (resp. the Hahn operator given by $\left.\left(H_{-1} f\right)(x)=\frac{f(x)-f(-x)}{2 x}\right) .{ }^{1}$

Y. Ben Cheikh and his coworker [1], the first who introduced the notion of Dunkl-classical orthogonal polynomials, derived a differential-difference equation for Dunkl-classical symmetric polynomials in order to prove that the only Dunkl-classical symmetric orthogonal polynomials are the generalized Hermite polynomials and the generalized Gegenbauer polynomials. Later on, M. Sghaier [15] characterized Dunkl-classical orthogonal polynomials by certain linear second order differential-difference equation. In such characterization, only the symmetric polynomials are considered.

The aim of this paper is to extend this characterization to nonsymmetric Dunkl-classical polynomials. Namely, we prove the following result.

Theorem 1.1. Let $\left\{P_{n}\right\}_{n \geq 0}$ a MOPS and let $u_{0}$ its associated form. The following assumptions are equivalent:

(a) The sequence $\left\{P_{n}\right\}_{n \geq 0}$ is Dunkl-classical.

(b) There exist a complex number $K$, a sequence $\left(\lambda_{n}\right)_{n \geq 0}$ in $\mathbb{C}^{*}$ and three polynomials $\Phi$ (monic), $\widetilde{\Phi}$ and $\Psi$ with $\operatorname{deg} \widetilde{\Phi}=\operatorname{deg} \Phi \leq 2$ and $\operatorname{deg} \Psi=1$ such that

$$
x \Phi(x) u_{0}=h_{-1}\left(x \widetilde{\Phi}(x) u_{0}\right)
$$

and

$$
\frac{K}{1-4 \mu^{2}}(\Phi(x)+2 \mu \widetilde{\Phi}(x))\left(T_{\mu}^{2} P_{n+1}\right)(x)-\Psi(x)\left(T_{\mu} P_{n+1}\right)(x)
$$

\footnotetext{
${ }^{1 \S}$ Corresponding author
} 


$$
-\frac{2 \mu K}{1-2 \mu}(\Phi(x)+\widetilde{\Phi}(x))\left(H_{-1} T_{\mu} P_{n+1}\right)(x)=\lambda_{n} P_{n+1}(x) .
$$

The structure of this paper is as follows: The second section is devoted to preliminary results and notations to be used in the sequel. In the third section, we prove the main theorem. In section 4, we illustrate results of the previous section by giving a differential-difference equation for a Dunkl-classical nonsymmetric polynomial sequence.

\section{Preliminaries and Notations}

Let $\mathcal{P}$ be the vector space of polynomials with coefficients in $\mathbb{C}$ and let $\mathcal{P}^{\prime}$ be its dual. We denote by $\langle u, f\rangle$ the action of $u \in \mathcal{P}^{\prime}$ on $f \in \mathcal{P}$. In particular, we denote by $(u)_{n}=\left\langle u, x^{n}\right\rangle, n \geq 0$ the moments of $u$.

The Dirac mass at the point $c \in \mathbb{C}$ denoted by $\delta_{c}$ is the form defined by

$$
\left\langle\delta_{c}, f\right\rangle=f(c), f \in \mathcal{P} .
$$

For $u \in \mathcal{P}^{\prime}$, we define the forms $h_{a} u, g u$ and $D u=u^{\prime}$ by:

$$
\begin{gathered}
\left\langle h_{a} u, f(x)\right\rangle=\left\langle u,\left(h_{a} f\right)(x)\right\rangle=\langle u, f(a x)\rangle, f \in \mathcal{P}, a \in \mathbb{C} \backslash\{0\}, \\
\langle g u, f\rangle=\langle u, g f\rangle,\left\langle u^{\prime}, f\right\rangle=-\left\langle u, f^{\prime}\right\rangle, f, g \in \mathcal{P} .
\end{gathered}
$$

Let $\left\{P_{n}\right\}_{n \geq 0}$ be a sequence of monic polynomials with $\operatorname{deg} P_{n}=n, n \geq 0$ and let $\left\{u_{n}\right\}_{n \geq 0}$ be its dual sequence, i.e. $u_{n} \in \mathcal{P}^{\prime}$ and defined by $\left\langle u_{n}, P_{m}\right\rangle=\delta_{n, m}$, where $\delta_{n, m}, n, m \geq 0$ is the kronecker delta function.

Let us recall some result [12]:

Lemma 2.1. For any $u \in \mathcal{P}^{\prime}$ and any integer $m \geq 1$, the following statements are equivalent:

(i) $<u, P_{m-1}>\neq 0,<u, P_{n}>=0, n \geq m$

(ii) $\exists \lambda_{\nu} \in \mathbb{C}, 0 \leq \nu \leq m-1, \lambda_{m-1} \neq 0$ such that $u=\sum_{\nu=0}^{m-1} \lambda_{\nu} u_{\nu}$.

The form $u$ is called regular if we can associate with it a PS $\left\{P_{n}\right\}_{n \geq 0}$ such that [4]:

$$
<u, P_{m} P_{n}>=r_{n} \delta_{n, m}, n, m \geq 0 ; r_{n} \neq 0, n \geq 0 .
$$

The sequence $\left\{P_{n}\right\}_{n \geq 0}$ is then said orthogonal with respect to $u$. In this case, we have $u_{n}=r_{n}^{-1} P_{n} u_{0}, n \geq 0$ and reciprocally. According to the previous 
lemma, we have $u=\lambda u_{0}$, where $(u)_{0}=\lambda \neq 0$. In what follows all regular forms $u$ will be taken normalized i.e, $(u)_{0}=1$.

According to Favard's theorem, a monic orthogonal polynomial sequence (MOPS, for shorter) $\left\{P_{n}\right\}_{n \geq 0}$ is characterized by the following three-term recurrence relation [4]:

$$
\begin{gathered}
P_{0}(x)=1, P_{1}(x)=x-\beta_{0}, \\
P_{n+2}(x)=\left(x-\beta_{n+1}\right) P_{n+1}(x)-\gamma_{n+1} P_{n}(x), n \geq 0 .
\end{gathered}
$$

A linear functional $u$ is said symmetric if and only if $(u)_{2 n+1}=0, n \geq 0$. Or equivalently, in (4) $\beta_{n}=0, n \geq 0$. Let us introduce the Dunkl's operator

$$
\left(T_{\mu} f\right)(x)=f^{\prime}(x)+2 \mu\left(H_{-1} f\right)(x), f \in \mathcal{P},
$$

where

$$
\left(H_{-1} f\right)(x)=\frac{f(x)-f(-x)}{2 x} .
$$

We define the operator $T_{\mu}$ from $\mathcal{P}^{\prime}$ to $\mathcal{P}^{\prime}$ as follows

$$
<T_{\mu} u, f>=-<u, T_{\mu} f>, f \in \mathcal{P}, u \in \mathcal{P}^{\prime} .
$$

In particular, this yields to

$$
\left(T_{\mu} u\right)_{n}=-\mu_{n}(u)_{n-1}, n \geq 0,
$$

where

$$
(u)_{-1}=0, \mu_{n}=n+2 \mu[n],[n]=\frac{1-(-1)^{n}}{2}, n \geq 0 .
$$

It is easy to see that

$$
T_{\mu} u=D u+2 \mu H_{-1} u,
$$

where

$$
<H_{-1} u, f>=-<u, H_{-1} f>.
$$

Now, consider a MOPS $\left\{P_{n}\right\}_{n \geq 0}$ as above and let

$$
P_{n}^{[1]}(x, \mu)=\frac{1}{\mu_{n+1}}\left(T_{\mu} P_{n+1}\right)(x), \mu \neq-n-\frac{3}{2}, n \geq 0 .
$$

Denoting by $\left\{u_{n}^{[1]}(\mu)\right\}_{n \geq 0}$ the dual sequence of $\left\{P_{n}^{[1]}(x, \mu)\right\}_{n \geq 0}$.

Lemma 2.2. [15]

$$
T_{\mu}\left(u_{n}^{[1]}(\mu)\right)=-\mu_{n+1} u_{n+1}, n \geq 0 .
$$




\section{Proof of the Main Theorem}

For the proof, we need the following lemma:

Lemma 3.1. [3] The following formula hold

$$
\begin{gathered}
T_{\mu} \delta_{0}=(1+2 \mu) \delta_{0}^{\prime}, \\
H_{-1} \delta_{0}=\delta_{0}^{\prime}, \\
H_{-1}\left(v-h_{-1} v\right)=0, v \in \mathcal{P}^{\prime}, \\
T_{\mu}(f u)=f T_{\mu} u+\left(T_{\mu} f\right) u+2 \mu\left(H_{-1} f\right)\left(h_{-1} u-u\right), f \in \mathcal{P}, u \in \mathcal{P}^{\prime},
\end{gathered}
$$

Proof of Theorem 1.1. First of all, notice that for $\mu=0$ we meet the D-classical orthogonal polynomial sequences, which satisfy a second order differential equation. Henceforth, we will suppose that $\mu \neq 0$.

$[(a) \Rightarrow(b)]$ From the assumption, we have

$$
u_{n}=r_{n}^{-1} P_{n} u_{0}, n \geq 0
$$

and

$$
u_{n}^{[1]}(\mu)=\left(r_{n}^{[1]}\right)^{-1} P_{n}^{[1]}(., \mu) u_{0}^{[1]}(\mu), n \geq 0 .
$$

Substitution of (10) and (11) in (5) gives

$$
T_{\mu}\left(P_{n}^{[1]}(., \mu) u_{0}^{[1]}(\mu)\right)=-\chi_{n} P_{n+1} u_{0}, n \geq 0,
$$

where

$$
\chi_{n}=\mu_{n+1} \frac{r_{n}^{[1]}}{r_{n+1}}, n \geq 0 .
$$

Using formula (9), equation (12) becomes

$$
\begin{aligned}
& P_{n}^{[1]}(., \mu) T_{\mu}\left(u_{0}^{[1]}(\mu)\right)+T_{\mu}\left(P_{n}^{[1]}(., \mu)\right) u_{0}^{[1]}(\mu)+ \\
&+2 \mu H_{-1} P_{n}^{[1]}(., \mu)\left(h_{-1} u_{0}^{[1]}(\mu)-u_{0}^{[1]}(\mu)\right)=-\chi_{n} P_{n+1} u_{0}, \\
& n \geq 0 .
\end{aligned}
$$

Putting $n=0$ in (14) and taking into account (13), we get

$$
T_{\mu}\left(u_{0}^{[1]}(\mu)\right)=-\Psi u_{0}
$$

where

$$
\Psi=\frac{1+2 \mu}{\gamma_{1}} P_{1}
$$


For $n=1$, equation (14) becomes

$$
P_{1}^{[1]}(., \mu) T_{\mu}\left(u_{0}^{[1]}(\mu)\right)+u_{0}^{[1]}(\mu)+2 \mu h_{-1} u_{0}^{[1]}(\mu)=-2 \frac{r_{1}^{[1]}}{r_{2}} P_{2} u_{0}
$$

Substitution of (15) in (17) gives

$$
u_{0}^{[1]}(\mu)+2 \mu h_{-1} u_{0}^{[1]}(\mu)=K \Phi u_{0},
$$

where

$$
K \Phi=\frac{1+2 \mu}{\gamma_{1}} P_{1} P_{1}^{[1]}(., \mu)-2 \frac{r_{1}^{[1]}}{r_{2}} P_{2},
$$

( $K$ is a constant to make $\Phi$ monic).

Applying the operator $h_{-1}$ to (18), we get

$$
2 \mu u_{0}^{[1]}(\mu)+h_{-1} u_{0}^{[1]}(\mu)=K h_{-1}\left(\Phi u_{0}\right) .
$$

Multiplication of (20) by $2 \mu$ and substraction of the result from (18) gives

$$
u_{0}^{[1]}(\mu)=\frac{K}{1-4 \mu^{2}}\left(\Phi u_{0}-2 \mu h_{-1}\left(\Phi u_{0}\right)\right) .
$$

Now, putting $n=2$ in (14), we obtain

$$
\begin{aligned}
P_{2}^{[1]}(., \mu) T_{\mu}\left(u_{0}^{[1]}(\mu)\right)+ & T_{\mu}\left(P_{2}^{[1]}(., \mu)\right) u_{0}^{[1]}(\mu)+ \\
& +2 \mu H_{-1} P_{2}^{[1]}(., \mu)\left(h_{-1} u_{0}^{[1]}(\mu)-u_{0}^{[1]}(\mu)\right)=-\chi_{2} P_{3} u_{0} .
\end{aligned}
$$

Taking into account (15) and (21), we get

$$
\begin{array}{r}
\frac{-2 \mu K}{1-4 \mu^{2}}\left(T_{\mu}\left(P_{2}^{[1]}(., \mu)\right)-(1+2 \mu) H_{-1} P_{2}^{[1]}(., \mu)\right) h_{-1}\left(\Phi u_{0}\right)= \\
\left(\frac{1+2 \mu}{\gamma_{1}} P_{1} P_{2}^{[1]}(., \mu)-\frac{K}{1-4 \mu^{2}} \Phi T_{\mu}\left(P_{2}^{[1]}(., \mu)\right)\right. \\
\left.+\frac{2 \mu K}{1-2 \mu} \Phi H_{-1} P_{2}^{[1]}(., \mu)-\chi_{2} P_{3}\right) u_{0} .
\end{array}
$$

Applying the operator $h_{-1}$ to the last equation and taking into account the facts that

$$
\begin{gathered}
h_{-1}(x v)=-x h_{-1} v, v \in \mathcal{P}^{\prime}, \\
h_{-1}\left(h_{-1} v\right)=v, v \in \mathcal{P}^{\prime}
\end{gathered}
$$


and

$$
T_{\mu}\left(P_{2}^{[1]}(., \mu)\right)-(1+2 \mu) H_{-1} P_{2}^{[1]}(., \mu)=2 x,
$$

we obtain

$$
x \Phi(x) u_{0}=h_{-1}\left(B(x) u_{0}\right),
$$

where

$$
\begin{array}{r}
B(x)=\frac{1-4 \mu^{2}}{4 \mu K}\left(\frac{1+2 \mu}{\gamma_{1}} P_{1} P_{2}^{[1]}(., \mu)-\frac{K}{1-4 \mu^{2}} \Phi T_{\mu}\left(P_{2}^{[1]}(., \mu)\right)\right. \\
\left.+\frac{2 \mu K}{1-2 \mu} \Phi H_{-1} P_{2}^{[1]}(., \mu)-\chi_{2} P_{3}\right) .
\end{array}
$$

To prove (1), we will show that in (23) polynomial $B$ can be written as:

$$
B(x)=x \widetilde{\Phi}(x), \widetilde{\Phi} \in \mathcal{P} .
$$

Using the definition of the operator $h_{-1}$, we can easily see that

$$
h_{-1}(f(x) v)=f(-x) h_{-1} v, f \in \mathcal{P}, v \in \mathcal{P}^{\prime} .
$$

Then, (23) can be written as

$$
x \Phi(x) u_{0}=B(-x) h_{-1} u_{0} .
$$

Application of $h_{-1}$ to $(26)$ gives

$$
B(x) u_{0}=-x \Phi(-x) h_{-1} u_{0} .
$$

Multiplying (26) and (27) by $-x \Phi(-x)$ and $B(-x)$ respectively and subtracting the result, we obtain

$$
-x^{2} \Phi(x) \Phi(-x) u_{0}=B(x) B(-x) u_{0} .
$$

By the regularity of $u_{0}$, we get

$$
-x^{2} \Phi(x) \Phi(-x)=B(x) B(-x) .
$$

Hence,

$$
B(0)=0, \operatorname{deg} B=1+\operatorname{deg} \Phi .
$$

Therefore, polynomial $B$ can be written as in (25), with $\operatorname{deg} \widetilde{\Phi}=\operatorname{deg} \Phi$ and, then, (1) follows immediately from (23). 
Now, multiplying (21) by $x$ and taking into account (23), we get

$$
x u_{0}^{[1]}(\mu)=\frac{K}{1-4 \mu^{2}}(x \Phi(x)+2 \mu B(x)) u_{0} .
$$

Applying the operator $h_{-1}$ to the last equation and using again (23), we obtain

$$
x h_{-1} u_{0}^{[1]}(\mu)=-\frac{K}{1-4 \mu^{2}}(B(x)+2 \mu x \Phi(x)) u_{0} .
$$

Multiplying (14) by $x$ and taking into account (15), (28) and (29), we get

$$
\begin{aligned}
\frac{K}{1-4 \mu^{2}}(x \Phi(x)+ & 2 \mu B(x)) T_{\mu} P_{n}^{[1]}(., \mu)-x \Psi(x) P_{n}^{[1]}(., \mu)+ \\
& -\frac{2 \mu K}{1-2 \mu}(x \Phi(x)+B(x)) H_{-1} P_{n}^{[1]}(., \mu)=-\chi_{n} x P_{n+1}(x),
\end{aligned}
$$

or, equivalently,

$$
\begin{aligned}
\frac{K}{1-4 \mu^{2}}(x \Phi(x) & +2 \mu B(x)) T_{\mu}^{2} P_{n+1}-x \Psi(x) T_{\mu} P_{n+1}+ \\
& -\frac{2 \mu K}{1-2 \mu}(x \Phi(x)+B(x)) H_{-1} T_{\mu} P_{n+1}=\lambda_{n} x P_{n+1}(x),
\end{aligned}
$$

where

$$
\lambda_{n}=-\chi_{n} \mu_{n+1} .
$$

Substitution of (25) in (31) and simplification by $x$ give (2).

$[(b) \Rightarrow(a)]$ Assume that equation (2) is fulfilled then using the definition of $\left\{P_{n}^{[1]}\right\}_{n \geq 0}$, we get

$$
\begin{aligned}
\frac{K}{1-4 \mu^{2}}(\Phi(x)+ & 2 \mu \widetilde{\Phi}(x))\left(T_{\mu} P_{n}^{[1]}\right)(x)-\Psi(x) P_{n}^{[1]}(x)+ \\
& -\frac{2 \mu K}{1-2 \mu}(\Phi(x)+\widetilde{\Phi}(x))\left(H_{-1} P_{n}^{[1]}\right)(x)=\frac{\lambda_{n}}{\mu_{n+1}} P_{n+1}(x) .
\end{aligned}
$$

Therefore,

$$
\begin{aligned}
0=\frac{\lambda_{n}}{\mu_{n+1}}<u_{0}, P_{n+1}>= & <u_{0}, \frac{K}{1-4 \mu^{2}}(\Phi(x)+2 \mu \widetilde{\Phi}(x)) T_{\mu} P_{n}^{[1]} \\
& -\Psi(x) P_{n}^{[1]}-\frac{2 \mu K}{1-2 \mu}(\Phi(x)+\widetilde{\Phi}(x)) H_{-1} P_{n}^{[1]}> \\
= & -<\frac{K}{1-4 \mu^{2}} T_{\mu}\left((\Phi(x)+2 \mu \widetilde{\Phi}(x)) u_{0}\right)+\Psi(x) u_{0} \\
& -\frac{2 \mu K}{1-2 \mu} H_{-1}((\Phi(x)+\widetilde{\Phi}(x))) u_{0}, P_{n}^{[1]}>, n \geq 0 .
\end{aligned}
$$


Taking into account the fact that $\left\{P_{n}^{[1]}(x)\right\}_{n \geq 0}$ is a basis sequence, we obtain

$$
\begin{aligned}
\frac{K}{1-4 \mu^{2}} T_{\mu}\left((\Phi(x)+2 \mu \widetilde{\Phi}(x)) u_{0}\right) & +\Psi(x) u_{0} \\
& -\frac{2 \mu K}{1-2 \mu} H_{-1}\left((\Phi(x)+\widetilde{\Phi}(x)) u_{0}\right)=0 .
\end{aligned}
$$

On the other hand, multiplying (23) by $x^{-1}$ and applying the formula

$$
x^{-1}(x v)=v-(v)_{0} \delta_{0}, v \in \mathcal{P}^{\prime},
$$

we get

$$
\Phi u_{0}=-h_{-1}\left(\widetilde{\Phi} u_{0}\right)+<u_{0}, \Phi(x)+\widetilde{\Phi}(x)>\delta_{0},
$$

or, equivalently,

$$
\widetilde{\Phi} u_{0}=-h_{-1}\left(\Phi u_{0}\right)+<u_{0}, \Phi(x)+\widetilde{\Phi}(x)>\delta_{0} .
$$

Substituting (35) in (33) and taking account of (6), (7) and (8) we obtain

$$
\frac{K}{1-4 \mu^{2}} T_{\mu}\left(\Phi u_{0}-2 \mu h_{-1}\left(\Phi u_{0}\right)\right)+\Psi u_{0}=0 \text {. }
$$

Therefore

$$
0=<\frac{K}{1-4 \mu^{2}} T_{\mu}\left(\Phi u_{0}-2 \mu h_{-1}\left(\Phi u_{0}\right)\right)+\Psi u_{0}, 1>=<u_{0}, \Psi>
$$

Taking into account that $\Psi$ is a polynomial of degree one, we get

$$
\Psi(x)=\lambda P_{1}(x), \lambda \neq 0 .
$$

So, (36) becomes

$$
T_{\mu}\left(\frac{K}{1-4 \mu^{2}}\left(\Phi u_{0}-2 \mu h_{-1}\left(\Phi u_{0}\right)\right)\right)=-\lambda P_{1}(x) u_{0} .
$$

But, according to Lemma 2.2,

$$
T_{\mu} u_{0}^{[1]}=-\frac{1+2 \mu}{\gamma_{1}} P_{1}(x) u_{0} .
$$

Therefore,

$$
T_{\mu} u_{0}^{[1]}=T_{\mu}\left(\frac{\alpha K}{1-4 \mu^{2}}\left(\Phi u_{0}-2 \mu h_{-1}\left(\Phi u_{0}\right)\right)\right)
$$


where

$$
\alpha=\frac{1+2 \mu}{\lambda \gamma_{1}} .
$$

Hence,

$$
u_{0}^{[1]}=\frac{\alpha K}{1-4 \mu^{2}}\left(\Phi u_{0}-2 \mu h_{-1}\left(\Phi u_{0}\right)\right) .
$$

Taking into account (23), we get

$$
\begin{gathered}
x u_{0}^{[1]}=\frac{\alpha K}{1-4 \mu^{2}}(x \Phi(x)+2 \mu B(x)) u_{0}, \\
x h_{-1} u_{0}^{[1]}(\mu)=-\frac{\alpha K}{1-4 \mu^{2}}(B(x)+2 \mu x \Phi(x)) u_{0} .
\end{gathered}
$$

Multiplying (32) by $\alpha x u_{0}$ and taking into account (37), (38), (39) and (23), we get

$$
x T_{\mu}\left(P_{n}^{[1]} u_{0}^{[1]}\right)=\alpha \frac{\lambda_{n}}{\mu_{n+1}} x P_{n+1} u_{0}, n \geq 0 .
$$

Using (34), we obtain

$$
T_{\mu}\left(P_{n}^{[1]} u_{0}^{[1]}\right)=\alpha \frac{\lambda_{n}}{\mu_{n+1}} P_{n+1} u_{0}, n \geq 0 .
$$

The regularity of $u_{0}^{[1]}$ comes from (3), (41) and the definition of $\left\{P_{n}^{[1]}\right\}_{n \geq 0}$ : For $n \geq m$ we have

$$
\begin{aligned}
<u_{0}^{[1]}, P_{m}^{[1]} P_{n}^{[1]}> & =-\frac{1}{\mu_{m+1}}<T_{\mu}\left(P_{n}^{[1]} u_{0}^{[1]}\right), P_{m+1}> \\
& =-\frac{\alpha \lambda_{n}}{\mu_{n+1} \mu_{m+1}}<P_{n+1} u_{0}, P_{m+1}> \\
& =-\frac{\alpha \lambda_{n}}{\mu_{n+1} \mu_{m+1}}<u_{0}, P_{m+1} P_{n+1}> \\
& =-\frac{\alpha \lambda_{n}}{\mu_{n+1}^{2}} r_{n} \delta_{n, m} .
\end{aligned}
$$

Therefore $\left\{P_{n}\right\}_{n \geq 0}$ is a Dunkl-classical orthogonal polynomial sequence.

Remark. The first author [3] showed that Dunkl-classical forms are characterized by (23) and (36) with the following condition:

$$
\Psi^{\prime}(0)+\frac{1}{2} \frac{K \Phi^{\prime \prime}(0)}{1-4 \mu^{2}}\left(4 \mu^{2}[n]-n\right)+\frac{1}{3} \frac{K B^{\prime \prime \prime}(0)}{\left(1-4 \mu^{2}\right)} \mu([n]-n) \neq 0 .
$$

In this paper, following the previous proof, we can easily see that from (23) and (36) only (without condition (42)) we prove the Dunkl-classical character. 
Corollary 3.2. A symmetric MOPS $\left\{P_{n}\right\}_{n \geq 0}$ is Dunkl-classical if and only if there exist a complex number $K$, a sequence $\left(\lambda_{n}\right)_{n \geq 0}$ in $\mathbb{C}^{*}$ and two polynomials $\Phi$ (monic), and $\Psi$ with $\operatorname{deg} \Phi \leq 2$ and $\operatorname{deg} \Psi=1$ such that

$$
\begin{aligned}
& \frac{K}{1-4 \mu^{2}}(\Phi(x)-2 \mu \Phi(-x))\left(T_{\mu}^{2} P_{n+1}\right)(x)-\Psi(x)\left(T_{\mu} P_{n+1}\right)(x)+ \\
& \quad-\frac{2 \mu K}{1-2 \mu}(\Phi(x)-\Phi(-x))\left(H_{-1} T_{\mu} P_{n+1}\right)(x)=\lambda_{n} P_{n+1}(x) .
\end{aligned}
$$

Proof. The sequence $\left\{P_{n}\right\}_{n \geq 0}$ is symmetric. Then, its corresponding form $u_{0}$ satisfies

$$
u_{0}=h_{-1} u_{0} .
$$

Multiplication of the above equation by $x \Phi(x)$ gives (1) with

$$
\widetilde{\Phi}(x)=-\Phi(-x) .
$$

Therefore, according to Theorem 1.1, $\left\{P_{n}\right\}_{n \geq 0}$ is a Dunkl-classical sequence if and only if it satisfies (2). Using (44), we can easily see that equation (2) is equivalent to (43). This completes the proof.

\section{Example}

In order to illustrate Theorem 1.1, we will consider the Dunkl-classical form $\widehat{\mathcal{G}}$ defined by:

$$
\widehat{\mathcal{G}}=(x-1) \mathcal{G}\left(\alpha, \mu-\frac{1}{2}\right),
$$

where $\mathcal{G}\left(\alpha, \mu-\frac{1}{2}\right)$ is the generalized Gegenbauer form.

This form is regular for $\alpha \neq 0, \mu+\frac{1}{2} \neq-n, n \geq 1$ and satisfies [15]

$$
T_{\mu}\left(\left(x^{2}-1\right) \widehat{\mathcal{G}}\right)+((-2 \alpha-2 \mu-3) x-(1+2 \mu)) \widehat{\mathcal{G}}=0 .
$$

The last equation is equivalent to (36), with

$$
\begin{aligned}
\Phi(x) & =(x+1)\left(x-\frac{1+2 \mu}{1-2 \mu}\right), \\
\Psi(x) & =\frac{(2 \alpha+2 \mu+3)^{2}}{2 \alpha+2}\left(x+\frac{1+2 \mu}{2 \alpha+2 \mu+3}\right), \\
K & =-\frac{(1-2 \mu)(2 \alpha+2 \mu+3)}{2 \alpha+2} .
\end{aligned}
$$


Furthermore, using (45) and the fact that $\mathcal{G}\left(\alpha, \mu-\frac{1}{2}\right)$ is symmetric, we can easily see that $\widehat{\mathcal{G}}$ satisfies (1) with

$$
\widetilde{\Phi}(x)=(x+1)\left(x+\frac{1+2 \mu}{1-2 \mu}\right) .
$$

Therefore, according to Theorem 1.1 and its proof, the MOPS corresponding to $\widehat{\mathcal{G}}$ satisfies

$$
\begin{aligned}
& \frac{K}{1-2 \mu}\left(x^{2}-1\right)\left(T_{\mu}^{2} P_{n+1}\right)(x)-\Psi(x)\left(T_{\mu} P_{n+1}\right)(x) \\
& -\frac{4 \mu K}{1-2 \mu} x(x+1)\left(H_{-1} T_{\mu} P_{n+1}\right)(x)=\lambda_{n} P_{n+1}(x),
\end{aligned}
$$

where $\lambda_{n}$ is a non-zero complex number.

Comparing the coefficients of higher degree of (47), we get

$$
\lambda_{n}=\frac{2 \alpha+2 \mu+3}{2 \alpha+2}\left(\mu_{n}-2 n-2 \alpha-2 \mu-3\right) \mu_{n+1} .
$$

Notes that $u_{0}$ is a nonsymmetric form. Since the sequence $\left\{P_{n}\right\}_{n \geq 0}$ satisfies (4) with [15]:

$$
\beta_{n}=a_{n+1}-a_{n}, n \geq 0
$$

where

$$
a_{2 n}=\frac{2 n+2 \alpha+2 \mu+1}{4 n+2 \alpha+2 \mu+1}, \quad a_{2 n+1}=\frac{2 n+2 \alpha+2}{4 n+2 \alpha+2 \mu+3}, \quad n \geq 0 .
$$

\section{Acknowledgments}

This work has been supported by Quassim University, Kingdom of Saoudi Arabia, grant 2638 .

\section{References}

[1] Y. Ben Cheikh, M. Gaied, Characterizations of the Dunkl-classical orthogonal polynomials, App. Math. Comput. 187 (2007), 105-114. http://dx.doi.org/10.1016/j.amc.2006.08.108

[2] S. Bochner, Uber Sturm-Liouvillesche Polynomsysteme, Math. Z. 29 (1929), 730736. http://dx.doi.org/10.1007/BF01180560 
[3] B. Bouras, Some characterizations of Dunkl-classical orthogonal polynomials, J. Difference. Equ. Appl. 20 (2014) 1240-1257. http://dx.doi.org/10.1080/10236198.2014.906590

[4] T. S. Chihara, An Introduction to Orthogonal Polynomials, Gordon and Breach, New York, (1978).

[5] C. F. Dunkl, Integral kernels reflection group invariance, Canad. J. Math. 43 (1991) 1213-1227. http://dx.doi.org/10.4153/CJM-1991-069-8

[6] A.G. Garcia, F. Marcellan, L. Salto, A distributional study of discrete classical orthogonal polynomials, J. Comput. Appl. Math. 57 (1995) 147162. http://dx.doi.org/10.1016/0377-0427(93)E0241-D

[7] L. Kheriji, P. Maroni, The Hq-classical orthogonal polynomials, Acta. Appl. Math. $\mathbf{7 1}$ (2002), 49-115. http://dx.doi.org/10.1023/A:1014597619994

[8] H. L. Krall and I. M. Sheffer, A characterization of orthogonal polynomials, J. Math. Anal. Appl. 8 (1964), 232244. http://dx.doi.org/10.1016/0022247X(64)90065-4

[9] K. H. Kwon and L. L. Littlejohn, Classification of classical orthogonal polynomials, J. Korean Math. Soc. 34 (1997), 9731008.

[10] K. H. Kwon, L. L. Littlejohn and B. H. Yoo, Characterizations of orthogonal polynomials satisfying differential equations, SIAM J. Math. Anal. 25, No. 3 (1994), 976990. http://dx.doi.org/10.1137/S0036141092236437

[11] L. L. Littlejohn, On the classification of differential equations having orthogonal polynomial solutions, Ann. Mat. Pura Appl. 4 (1984), 3553. http://dx.doi.org/10.1007/BF01762538

[12] F. Marcellán, A. Branquinho, J. Petronilho, Classical orthogonal polynomials: A functional approach, Acta. Appl. Math. 34 (1994), 283-303. http://dx.doi.org/10.1007/BF00998681

[13] P. Maroni, Variation around Classical orthogonal polynomials. Connected problems, J. Comput. Appl. Math. 48 (1993), 133-155. http://dx.doi.org/10.1016/0377-0427(93)90319-7

[14] J.C. Medem, R. Alvarez-Nodarse, F. Marcellan, On the q-polynomials: A distributional study, J. Comput. Anal. Math. 135 (2001) 157196. http://dx.doi.org/10.1016/S0377-0427(00)00584-7 
[15] M. Sghaier, A note on Dunkl-classical orthogonal polynomials, Integral. Transforms. Spec. Funct. 24 (2012), $753-760$. http://dx.doi.org/10.1080/10652469.2011.631186 